\title{
Schwinger boson theory of anisotropic ferromagnetic ultrathin films
}

\author{
Carsten Timm 1 and P.J. Jensen ${ }^{2,1}$ \\ ${ }^{1}$ Institut für Theoretische Physik, Freie Universität Berlin, Arnimallee 14, D-14195 Berlin, Germany \\ ${ }^{2}$ Hahn-Meitner-Institut Berlin, Glienicker Str. 100, D-14109 Berlin, Germany
}

(December 21, 1999)

\begin{abstract}
Ferromagnetic thin films with magnetic single-ion anisotropies are studied within the framework of Schwinger bosonization of a quantum Heisenberg model. Two alternative bosonizations are discussed. We show that qualitatively correct results are obtained even at the mean-field level of the theory, similar to Schwinger boson results for other magnetic systems. In particular, the MerminWagner theorem is satisfied: a spontaneous magnetization at finite temperatures is not found if the ground state of the anisotropic system exhibits a continuous degeneracy. We calculate the magnetization and effective anisotropies as functions of exchange interaction, magnetic anisotropies, external magnetic field, and temperature for arbitrary values of the spin quantum number. Magnetic reorientation transitions and effective anisotropies are discussed. The results obtained by Schwinger boson mean-field theory are compared with the many-body Green's-function technique.
\end{abstract}

PACS numbers: 75.10.Jm, 75.30.Gw, 75.70.Ak

\section{INTRODUCTION}

Schwinger boson theories are very successful indescribing magnetism in various quantum systems.t-1 Qualitatively correct results are mostly obtained even in the mean-field approximation, e.g., quantum Heisenberg ferromagnets and antiferromagnets are well described for arbitrary spin quantum number for two and more spatial dimensions. In particular in two-dimensional (2D) isotropic Heisenberg magnets no spontaneous magnetization is found for non-zero temperatures 1 由 in accordance with the Mermin-Wagner theorem. There are basically two reasons why Schwinger boson mean-field (SBMF) theory works well even in low dimensions: (a) Since the bosonized spin degrees of freedom are integrated over in the functional integral, spin fluctuations are taken into account and (b) the approach does not constitute an expansion around an ordered state, unlike Holstein-Primakov bosonization, 1 and thus works for both ordered and disordered ground states.

The first aim of the present paper is to test the applicability of SBMF theory to another class of quantummagnetic models, namely the Heisenberg ferromagnet with single-ion (on-site) anisotropies. We will restrict ourselves to the two-dimensional case, which is the most interesting one, but the same methods can be used in any number of dimensions. The anisotropic Heisenberg model in two dimensions is of particular interest, since it describes ultra-thin ferromagnetic films, including the important cases of the $3 d$ transition metals iron, cobalt, and nickel. Thin ferromagnetic films are of great technological relevance, e.g., for magnetic disk drives. They also show many interesting physical effects, of which we only mention the observed reorientation transitions-as a function of both film thickness and temperature.10 00 Much theoretical work has already been done on anisotropic films, which we briefly review below. However, it would be desirable to have an alternative approach for comparison. The second aim of this paper is to provide such an approach using Schwinger bosonization. We apply this method to the calculation of the magnetization with and without an external magnetic field. In addition we determine effective anisotropies, which are crucial for comparisons of measurements performed at finite temperatures $T$ to ab-initio calculations of anisotropies at $T=0$. The effective anisotropies describe the dependence of the free energy on the polar angles $(\theta, \phi)$ of the magnetization.

From the theoretical point of view, two-dimensional anisotropic ferromagnets are of particular interest, since the anisotropies reduce the symmetry of the system. For example, an easy axis leads to a discrete, Ising-type symmetry, while a easy plane leads to an $X Y$-typ symmetry. According to the Mermin-Wagner theorem, neous magnetization at finite temperatures is possible in the first case but not in the second.21 We will show that SBMF theory yields qualitatively correct results for both of these cases.

We now summarize previous work on thin magnetic films. So far, most approaches rely on simple mean-field approximations, which are of limited validity for lowdimensional systems. In particular, they do not satisfy the Mermin-Wagner theorem. A finitemagnetization can be induced by magnetic anisotropies, 21 which, however, cannot be described by a perturbative expansion around the symmetric state. Thus, improved approaches are needed, which should also allow to determine the magnetic properties in the whole range of temperatures and for systems with nonequivalent lattice sites. For example, magnetic reorientation transitions have been studied within spin-wave theories. In particular HolsteinPrimakov bosonization 8 has been applied 22 which is, however, limited to low temperatures.

The magnetic properties of $2 \mathrm{D}$ anisotropic ferromagnets have also been studied using many-body Green's functions, which allow calculations in the whole temperature range of interest.23 27 This methad has also been applied to thin films of several layers. 2833 Within this theory the higher-order Green's functions are approxi- 
mated by the so-called Twablicov (or random-phase approximation) decoupling 23 i.e., one of the spin operator components is replaced by its expectation value, whereas the expectation values of the other two components vanishes. Note that within this approach the spin commutation relations are preserved. By comparison with a recent Quantum-Monte-Carlo calculation for an isotropic 2D magnet with $S=1 / 2$ on a square lattice in an external magnetic field, 4 it has been shown that this approach_vields a reasonable description for the magnetization.35 In addition, the long-range magnetic dipole interaction can be treated as well within the same decoupling scheme. On the other hand, single-ion anisotropies have to be treated differently, since the inclusion of such terms leads to an ambigous description. 36 Anderson and Callen, 37 as well as Lines 38 have proposed interpolation schemes, which are valid only for small single-ion interactions as compared to the exchange coupling. For most cases of interest this condition is fulfilled. In order to calculate the effective anisotropies $\mathcal{K}_{i}(T)$ and the temperature-dependent magnetic reorientation, nonvanishing expectation values of at least two spin opera tor components have to be determined simultaneously.27 Whereas the inclusion of the magnetic dipole coupling is straightforward, the inclusion of single-ion anisotropies turns out to be tedious, in particular for spin quantum numbers $S>1$, and for higher-order anisotropies. We emphasize that within these Green's-function approaches the magnetic anisotropies cannot be handled as small perturbations. Also, this approach suffers from the ambigous decoupling schemes for these single-ion terms. Thus, a theory not suffering from these problems would be desirable.

Finally, we summarize other approaches. First, meanfield theories have been used,394 which are particularly simple to apply for complicated systems, e.g., with nonequivalent atomic layers in a thin film or several nonequivalent lattice sites. Classical as well as quantummechanical Heisenberg spins have been studied allowing for an arbitrary direction of the magnetization. The inclusion of single-ion anisotropies is quite simple. A perturbative treatment of the anisotropies yields analytical expressions for the effective anisotropies. $45,42,40$ However, these approaches completely neglect both thermal and quantum fluctuations, which are crucial for lowdimensional systems. Kawazoe et al. 46 have treated the magnetic reorientation of thin ferromagnetic films within a continuum approach for the system and mean-fieldlike expressions for the magnetization density. A continuum approach to the magnetic reorientation has also been used within a renormalization-group treatment. 47

On the other hand, following earlier work 48 , te Millev et al 49 treat the magnetization of a thin film as a macroscopic, classical magnetic moment and discuss its ground state as a function of anisotropy and external magnetic field. The anisotropy constants appearing in their work are to be understood as effective anisotropies obtained from experiment or from some more advanced calcula- tion. This approach has to assume that the effects of thermal and quantum fluctuations, the lattice structure, and the spin quantum number $S$ can be described by a suitable renormalization of the anisotropy constants. From a theoretical point of view, the problem has just been transferred to the calculation of these effective anisotropies. This has to be done using more advanced methods such as Schwinger bosonization (discussed below) or many-body Green's functions.

Finally, Monte-Carlo simulations have been performed for the effective anisotropies and magnetic reorientation of ferromagnetic monolayers. So far, only flassical spins have been studied for the anisotropic case, 50 while Quantum-Monte-Carlo results exist for the isotropic case only. 34 More complicated systems such as thin films with several layers or including the dipole interaction have not been studied yet.

Following the goals set above, we derive in Sec. II SBMF theories for films with second- and fourth-order single-ion anisotropy. We employ two different bosonization schemes, known as $\mathrm{SU}(N)$ and $\mathrm{O}(N)$ bosonization. In Sec. III we show representative results and compare them with results from simple mean-field theories and from many-body Green's-function methods. We focus on two areas of current interest: In Sec. III A we discuss the change of magnetization as a function of external field and of temperature, in particular the reorientation transitions, and present a number of phase diagrams. In Sec. IIIB we calculate temperature-dependent effective anisotropies. A summary and conclusions are given in Sec. IV.

\section{SCHWINGER BOSON THEORY}

In this section we summarize the SBMF theory for an anisotropic 2D ferromagnet, using two different, but related, bosonizations. The first one employs the $\mathrm{SU}(2)$ symmetry of the spind 1 in the absence of anisotropies and leads to an $\mathrm{SU}(N)$-symmetric mean-field theory, where $N \rightarrow \infty$. The second makes use of the mapping between the Lie groups $\mathrm{S} U(2)$ and $\mathrm{O}(3)$ and results in an $\mathrm{O}(N)$ mean-field theory. 6 We first sketch the $\mathrm{SU}(N)$ theory, which is described in greater detail in App. A. The derivation for the $\mathrm{O}(N)$ theory is similar and we only present the results.

The main idea of $\mathrm{SU}(2)$ Schwinger boson theory is to map the spippperators onto boson operators $b_{\uparrow}$ and $b_{\downarrow}$ according toll

$$
\begin{aligned}
S^{+} & =b_{\uparrow}^{\dagger} b_{\downarrow}, \\
S^{-} & =b_{\downarrow}^{\dagger} b_{\uparrow}, \\
S^{z} & =\left(b_{\uparrow}^{\dagger} b_{\uparrow}-b_{\downarrow}^{\dagger} b_{\downarrow}\right) / 2 .
\end{aligned}
$$

This mapping is exact; in particular the spin commutation relations are correctly reproduced. However, the 
bosonization introduces unphysical degrees of freedom, which have to be removed by the constraint

$$
b_{\uparrow}^{\dagger} b_{\uparrow}+b_{\downarrow}^{\dagger} b_{\downarrow}=2 S,
$$

where $S$ is the spin quantum number. Whereas the above scheme employs the $\mathrm{SU}(2)$ spin symmetry group, one can also take advantage of the mapping between $\mathrm{SU}(2)$ and the group $\mathrm{O}(3)$ - both have the same algebra up to the choice of representation - to farmulate an alternative $\mathrm{O}(3)$ Schwinger boson theory. 6 For both groups well-defined expansions around a mean-field theory are obtained by generalization to $N$ boson fields and expanding in small $1 / N$. In particular, in the limit $N \rightarrow \infty$ mean-field theory becemes exact.

Read and Sachdev 6 have derived $\mathrm{SU}(N)$ and $\mathrm{O}(N)$ SBMF expressions for the magnetization of isotropic 2D ferromagnets, where their main interest was in thequantum Hall ferromagnet at filling factor $\nu=1.51 .52$ Recently, the leading-order fluctuation corrections, i.e., the first order of the $1 / N$ expansion, have been calculated and compared to Quantym-Monte-Carlo simulations 34 , and experimental results.53 The $\mathrm{SU}(N)$ and $\mathrm{O}(N)$ theories are found to be qualitatively correct. In the quantum Hall system the magnetic degrees of freedom are carried by conduction electrons with $S \overline{1}^{1 / 2}$. Thus single-ion anisotropies do not play any role. 4

We describe the anisotropic 2D ferromagnet by a Heisenberg model on a 2D square lattice, augmented by second- and fourth-order single-ion anisotropies,

$$
\begin{aligned}
H= & -J \sum_{\langle i j\rangle} \mathbf{S}_{i} \cdot \mathbf{S}_{j}-\mathbf{B} \cdot \sum_{i} \mathbf{S}_{i}-K_{2} \sum_{i}\left(S_{i}^{z}\right)^{2} \\
& -K_{4}^{\perp} \sum_{i}\left(S_{i}^{z}\right)^{4}-K_{4}^{\|} \sum_{i}\left[\left(S_{i}^{x}\right)^{4}+\left(S_{i}^{y}\right)^{4}\right],
\end{aligned}
$$

where $\mathbf{S} \cdot \mathbf{S}=S(S+1)$ and the sum over $\langle i j\rangle$ is over nearest neighbors, counting each bond once. $J$ is the exchange constant and $\mathbf{B}$ is the external magnetic field. Positive anisotropy parameters $K_{2}, K_{4}^{\perp}>0$ favor a perpendicular magnetization (easy-axis magnet), while the opposite sign favors an in-plane magnetization (easy-plane magnet). Two important special cases are $K_{4}^{\|}=0$, corresponding to uniaxial symmetry, and $K_{2}=0, K_{4}^{\|}=K_{4}^{\perp}$, corresponding to cubic symmetry. Equation (5) is viewed as a microscopic Hamiltonian for a ferromagnetic monolayer, where the anisotropy parameters are obtained, for example, from first-principles calculations. The same Hamiltonian can also be used approximately as an effective model for films with several layers, in which case the anisotropy constants are to be understood as averaged parameters containing both bulk and surface contributions. Since anisotropy energies (and also the exchange coupling $J$ ) can be quite different for the surface layers and for the bulk, the effective parameters can be tuned by changing the film thickness.

Equation (5) does not include the magnetic dipolar interaction

$$
H_{\text {dip }}=\frac{\omega}{2} \sum_{i, j(i \neq j)}\left[\frac{\mathbf{S}_{i} \cdot \mathbf{S}_{j}}{r_{i j}^{3}}-\frac{3\left(\mathbf{S}_{i} \cdot \mathbf{r}_{i j}\right)\left(\mathbf{S}_{j} \cdot \mathbf{r}_{i j}\right)}{r_{i j}^{5}}\right],
$$

where $\mathbf{r}_{i j}=\mathbf{r}_{i}-\mathbf{r}_{j}$ is the separation vector of sites $i$ and $j, \omega=\mu_{0} g^{2} \mu_{B}^{2} / 4 \pi, \mu_{0}$ is the permeability of free space, $g$ is the Landé factor, and $\mu_{B}$ is the Bohr magneton. The dipolar interaction is difficult to treat in the present framework, mainly because of its long-range nature. One may incorporate it in a simple mean-field way by replacing one of the two spin operators in Eq. (6) by its thermal average, which is, in our notation, the magnetization $\mathbf{M}$. In a continuum approximation this leads to

$$
H_{\text {dip }} \cong-\frac{1}{2} \frac{2 \pi \omega}{3 a^{3}}\left(M_{x}, M_{y},-2 M_{z}\right) \cdot \sum_{i} \mathbf{S}_{i}
$$

where $a$ is the lattice constant. Equation (7) describes the interaction with an effective demagnetizing field. This term explicitly breaks spin symmetry in that it prefers the magnetization to lie in the $x y$ plane, as is well known. In this approximation, the rotational symmetry within the plane is retained. A better description would also break this residual symmetry. This is the reason why a magnetic monolayer has a finite in-plane magnetization even if only the exchange coupling and the magnetic dipole interaction are considered as has been shown using a Green's-function approach.22 27 Since we are mainly interested in the effects of the single-ion anisotropy terms in the Hamiltonian and in spontaneous symmetry breaking, we neglect $H_{\text {dip }}$ for most of this paper.

We now give an overview over the derivation: After replacing the spin operators in the Hamiltonian (5) by bosons, the bosonic system is generalized from two to $N$ boson species. We mention that we take care to preserve the quantum properties of the spin operators in the anisotropy terms as we perform the limit $N \rightarrow \infty$. Then the partition function $Z$ is written as a functional integral over complex auxilliary fields. The constraint on boson number is incorporated using a Lagrange-multiplier field and the exchange interaction as well as the anisotropy terms are decoupled by means of a Hubbard-Stratonovich transformation. Within the mean-field approximation the auxilliary fields are then replaced by constants, which have to be obtained from saddle-point equations.

The resulting mean-field free energy $F_{0}$ depends on the four auxilliary parameters $\overline{\mathbf{P}}$ and $\bar{\Lambda}$. One has to find the saddle points of $F_{0}$ with respect to these parameters and check their stability, as discussed in App. A. $\bar{\Lambda}$ can be interpreted as a chemical potential that globally enforces the constraint on boson number, whereas the vector $\overline{\mathbf{P}}$ is related to the magnetization see below. Introducing rescaled anisotropy parameters 55

$$
\begin{aligned}
\tilde{K}_{2} & =K_{2} \frac{S-1 / 2}{S}, \\
\tilde{K}_{4}^{\perp, \|} & =K_{4}^{\perp, \|} \frac{(S-1 / 2)(S-1)(S-3 / 2)}{S^{3}}
\end{aligned}
$$


and the inverse temperature $\beta=1 / k_{B} T$, the free energy reads

$$
\begin{aligned}
\beta F_{0}= & -S \bar{\Lambda}+\frac{\beta \tilde{K}_{2}}{2} \bar{P}_{z}^{2} \\
& +\frac{3 \beta \tilde{K}_{4}^{\perp}}{2} \bar{P}_{z}^{4}+\frac{3 \beta \tilde{K}_{4}^{\|}}{2}\left(\bar{P}_{x}^{4}+\bar{P}_{y}^{4}\right) \\
& +\frac{1}{8 \pi \beta J S}\left[\operatorname{diln}\left(1-e^{-\bar{\Lambda}+\beta B^{\prime} / 2}\right)\right. \\
& \left.+\operatorname{diln}\left(1-e^{-\bar{\Lambda}-\beta B^{\prime} / 2}\right)\right]
\end{aligned}
$$

with the dilogarithm function diln and the effective magnetic field

$$
\begin{aligned}
& B_{x}^{\prime}=B_{x}+4 \tilde{K}_{4}^{\|} \bar{P}_{x}^{3}, \\
& B_{y}^{\prime}=B_{y}+4 \tilde{K}_{4}^{\|} \bar{P}_{y}^{3}, \\
& B_{z}^{\prime}=B_{z}+2 \tilde{K}_{2} \bar{P}_{z}+4 \tilde{K}_{4}^{\perp} \bar{P}_{z}^{3} .
\end{aligned}
$$

We emphasize that the whole mathematical apparatus of $\mathrm{SU}(N)$ SBMF theory is contained in Eqs. (10)-(14) Note that these expressions reduce to the isotropic case 6 - for $K_{2}=K_{4}^{\perp}=K_{4}^{\|}=0$ and that the second-order (fourthorder) anisotropies only enter the results if $S \geq 1(S \geq 2)$.

The mean-field magnetization is given by

$$
\begin{aligned}
& \mathbf{M}_{0} \equiv-2 \frac{d F_{0}}{d \mathbf{B}}=-\frac{1}{8 \pi \beta J S} \frac{\mathbf{B}^{\prime}}{B^{\prime}} \\
& \quad \times\left[\ln \left(1-e^{-\bar{\Lambda}+\beta B^{\prime} / 2}\right)-\ln \left(1-e^{-\bar{\Lambda}-\beta B^{\prime} / 2}\right)\right],
\end{aligned}
$$

where $B^{\prime}=\left|\mathbf{B}^{\prime}\right|$. As shown in App. A, $\bar{P}_{i}$ as obtained from the saddle-point equations equals the magnetization component $M_{0, i}$, if the free energy $F_{0}$ depends on $\bar{P}_{i}$. On the other hand, if $F_{0}$ does not depend on $\bar{P}_{i}$, the latter quantity can assume any value, while the magnetization $M_{0, i}$ still has a well-defined value given by Eq. (14).

Within the simplified treatment of the dipolar interaction discussed above, a demagnetizing field proportional to $\left(M_{0, x}, M_{0, y},-2 M_{0, z}\right)$ would be added to $\mathbf{B}^{\prime}$. By using a Green's-function method it can be shown that this yields an acceptable result for the magnetization if the magnetic dipole fqupling is small as compared to the exchange coupling.56

Finally, we give the results of $\mathrm{O}(N)$ SBMF theory without derivation. The free energy per boson reads

$$
\begin{aligned}
& \beta F_{0}^{\mathrm{O}(N)}=-\frac{1}{3} S \bar{\Lambda}+3 \beta \tilde{K}_{2} \bar{P}_{z}^{2} \\
& \quad+81 \beta \tilde{K}_{4}^{\perp} \bar{P}_{z}^{4}+81 \beta \tilde{K}_{4}^{\|}\left(\bar{P}_{x}^{4}+\bar{P}_{y}^{4}\right) \\
& +\frac{1}{12 \pi \beta J S}\left[\operatorname{diln}\left(1-e^{-\bar{\Lambda}+\beta B^{\prime}}\right)\right. \\
& \left.\quad+\operatorname{diln}\left(1-e^{-\bar{\Lambda}}\right)+\operatorname{diln}\left(1-e^{-\bar{\Lambda}-\beta B^{\prime}}\right)\right]
\end{aligned}
$$

with the effective field

$$
\begin{aligned}
& B_{x}^{\prime}=B_{x}+108 \tilde{K}_{4}^{\|} \bar{P}_{x}^{3}, \\
& B_{y}^{\prime}=B_{y}+108 \tilde{K}_{4}^{\|} \bar{P}_{y}^{3}, \\
& B_{z}^{\prime}=B_{z}+6 \tilde{K}_{2} \bar{P}_{z}+108 \tilde{K}_{4}^{\|} \bar{P}_{z}^{3},
\end{aligned}
$$

and the magnetization is

$$
\begin{aligned}
& \mathbf{M}_{0}^{\mathrm{O}(N)}=-\frac{1}{4 \pi \beta J S} \frac{\mathbf{B}^{\prime}}{B^{\prime}} \\
& \quad \times\left[\ln \left(1-e^{-\bar{\Lambda}+\beta B^{\prime}}\right)-\ln \left(1-e^{-\bar{\Lambda}-\beta B^{\prime}}\right)\right] .
\end{aligned}
$$

The general form of these expressions is similar to the $\mathrm{SU}(N)$ case and we thus expect qualitatively similar results.

\section{RESULTS AND DISCUSSION}

In this section we discuss a number of results obtained from SBMF theory and compare them with other approaches. Our aim is mainly to show that SBMF theory yields qualitatively correct results in the presence of single-ion anisotropies. We further show that these results are comparable to Green's-function methods, although they are much easier to obtain. We first discuss the magnetization $M_{0}$ and then effective anisotropies.

\section{A. Magnetization}

SBMF theory correctly predicts the magnetization of an isotropic 2D Heisenberg ferromagnet to vanish at finite temperatures in the absence of an external magnetic field. 6 However, an easy-axis uniaxial anisotropy $K_{2}>0$ explicitly breaks the $\mathrm{SU}(2)$ spin symmetry down to a residual Ising symmetry. A discrete symmetry can be spontaneously broken and one expects a finitetemperature phase transition between an ordered and a disordered phase. Both $\mathrm{SU}(N)$ and $\mathrm{O}(N)$ theory indeed yield a finite $T_{c}$. Figure 11 shows the magnetization of a spin-1 system with a second-order uniaxial anisotropy $K_{2} / J=0.01$ in the absence of an external magnetic field, as a function of the reduced temperature $T / T_{c}$. As usual, simple mean-field theory overestimates the magnetization. In particular, at low temperatures the magnetization approaches $\mathbf{M}(T=0)$ exponentially due to the neglect of spin waves. On the other hand, $\mathrm{SU}(N)$ and $\mathrm{O}(N)$ Schwinger beson theories and many-body Green'sfunction method\$27 correctly predict an almost linear behavior. However, the absolute temperature scales are rather different, as shown in the inset of Fig. 1. Numerical results, e.g., from Quantum-Monte-Carlo simulations, would be very useful to check the absolute value of $T_{c}$.

One can obtain analytical expressions for $T_{c}$ by expanding the saddle-point equtions for a small magnetization $M_{0, z}$. The results are 57 


$$
T_{c}^{\mathrm{SU}(N)}=\frac{4 \pi J S^{2}}{\ln \left[1+4 \pi J S^{2} / K_{2}(S-1 / 2)\right]}
$$

for $\mathrm{SU}(N)$ theory and

$$
T_{c}^{\mathrm{O}(N)}=\frac{4 \pi J S^{2}}{3 \ln \left[1+\pi J S^{2} / K_{2}(S-1 / 2)\right]}
$$

for $\mathrm{O}(N)$ theory. Both expressions have the same functional form. In particular, $T_{c}$ vanishes in the limit $K_{2} \rightarrow 0^{+}$, as it should, and $T_{c}=0$ for $S=1 / 2$. For comparison, using a continuum approximation similar to the one employed here, the many-body Green's-function method yields

$$
T_{c}^{\mathrm{RPA}}=\frac{4 \pi J S(S+1)}{3 \ln \left[1+3 \pi^{2} J S / 4 K_{2}(S-1 / 2)\right]}
$$

within the Anderson-Callen decoupling of the secondorder anisotropy. 37 Note that all three expressions show the logarithmic dependence of $T_{c}$ on $K_{2}$, while the dependence on the spin quantum number differs.

Since the results of $\mathrm{SU}(N)$ and $\mathrm{O}(N)$ theory qualitatively agree with each other, we concentrate on $\mathrm{SU}(N)$ in the following. In the case of a hard axis, $K_{2}<0$, and $K_{4}^{\|}=0$, the residual symmetry is of $X Y$ type. Because of the continuous degeneracy there should be no spontaneous magnetization. This is indeed found within SBMF theory. If we include a dipolar interaction by means of Eq. (7), a finite in-plane magnetization emerges, since in this approximation the dipole coupling acts similarly to an external field.

In the presence of an external magnetic field $\mathbf{B}$ one always expects a finite magnetization. For an isotropic film this magnetization is parallel to the applied field, whereas in the presence of anisotropy the magnetization may be oriented along a different direction. In addition, a magnetic reorientation transition can occur, which may be controlled by varying the temperature $T$, the external magnetic field $\mathbf{B}$, or the film thickness. In Fig. 2(a) the magnetization components parallel and perpendicular to the magnetic field are shown as functions of its strength. The field is applied perpendicularly to the easy axis, which is realized by a second-order uniaxial anisotropy $K_{2} / J=0.01$. Within our theory we find a linear increase of the in-plane magnetization along the magnetic-field direction below a critical reorientation field $B_{\text {reo }}$. Since the modulus of the magnetization is almost constant, the perpendicular magnetization component decreases correspondingly. At $B=B_{\text {reo }}$ the latter component vanishes continuously, corresponding to a second-order phase transition. For $B \geq B_{\text {reo }}$ the magnetization is parallel to the field and increases only weakly with $B$. Figure 2(b) shows a similar situation, with the easy axis realized by the fourth-order uniaxial anisotropy $K_{4}^{\perp}>0$, while $K_{2}=K_{4}^{\|}=0$. Again a reorientation transition is obtained as a function of the in-plane magnetic field. However, since here the magnetization changes discontinuously, the transition is of first order. We expect that the dependence of the order of the transition on the order of the uniaxial anisotropy is a universal feature, since the same behavior has been observed within simpler theories. 88.4

To show the interplay of the various interaction terms, we now discuss several phase diagrams. For comparison, Fig. 3 shows the well-known phase diagram in the $\mathcal{K}_{2}-\mathcal{K}_{4}^{\perp}$ plane for $\mathcal{K}_{4}^{\|}=0$ and $\mathbf{B}=0$ derived under the assumption that the magnetization pepres like a macroscopic, classical magnetic moment. 180.49 The anisotropies should be viewed as effective quantities, which depend on temperature etc. Roughly, a perpendicular magnetization is found for $\mathcal{K}_{2}>0$ and $\mathcal{K}_{4}^{\perp}>0$, 99 an in-plane one for $\mathcal{K}_{2}<0$ and $\mathcal{K}_{4}^{\perp}<0$, and a canted one for $\mathcal{K}_{2}>0$ and $\mathcal{K}_{4}^{\perp}<0$. These magnetic phases are separated by continuous (second order) or discontinuous (first order) phase transition boundaries, which merge in a tricritical point. A coexistence region between the perpendicular and the in-plane magnetization is located close to the first-order phase transition line. As usual, the first-order phase boundary has been drawn where the free energies of both phases are equal.

The simple phase diagram of Fig. 3 does not apply to $2 \mathrm{D}$ systems, since there should be no net in-plane magnetization if solely uniaxial anisotropies are taken into account. The reason is that in this case a ground state with finite in-plane magnetization would be continuously degenerate 3 The origin of this problem of course lies in the neglect of fluctuations.

This picture is changed if fluctuations are included by means of SU(N) SBMF theory: Figure 1 shows the phase diagram in the $K_{2}-K_{4}^{\perp}$ plane for a thin film at a finite temperature $T / J=3$ for spin $S=260$ The anisotropy parameters are now the microscopic ones contained in the Hamiltonian (5). Note that the scale of the anisotropies in Fig. 1 is small, since the temperature $T / J=3$ is significantly lower than the typical temperature scale $4 \pi J S^{2}$ of Eq. (20). This means that very small anisotropies are sufficient to induce a finite magnetization, since $K_{2}$ enters logarithmically in $T_{c}$, Eq. (20).

In Fig. 1 only two phases are present: A phase without net magnetization located approximately in the region $K_{2}<0$ and $K_{4}^{\perp}<0$ and a phase with perpendicular magnetization otherwise. These two phases are separated by either a first-order or a second-order transition. The tricritical point, at which the two merge, has shifted to finite, temperature-dependend values of the anisotropies $K_{2 c}>0$ and $K_{4 c}^{\perp}>0$. For smaller anisotropies thermal fluctuations overcome the tendency to order. A coexistence region is found in the vicinity of the first-order transition. The first-order line is no longer straight and approaches the tricritical point with a vertical tangent. For negative $K_{4}^{\perp}$ the second-order transition line is given by $K_{2}=$ const, similar to Fig. 3. Thus the location of this transition depends only on $K_{2}$. At this transition the zero-magnetization $(\mathbf{M}=0)$ saddle point becomes unstable. The free energy for small $\mathbf{M}$, and thus the sta- 
bility of this saddle point, is solely determined by the lowest-order anisotropy $K_{2}$.

In-plane and canted magnetic phases do not appear in Fig. 4, since this system is either in the Ising or in the $X Y$ universality class. However, such phases do exist if the in-plane symmetry is explicitely broken, e.g., by a single-ion in-plane anisotropy $K_{4}^{\|} \neq 0$, by an external magnetic field, or by the dipole interaction. Here we consider a small fourth-order in-plane anisotropy $K_{4}^{\|} / J \approx 4.3 \times 10^{-4}>0$. The other parameters are unchanged. The resulting phase diagram is shown in Fig. 5, exhibiting now three phases similar to Fig. 3. However, the transition between the canted and perpendicular phases is now of first order (the first-order line extends to arbitrarily large negative $\left.K_{4}^{\perp}\right)$. The first-order character is typically weak - the jump in the magnetization is rather small. The coexistence regions are also shown in Fig. 5. Note that for even smaller $K_{4}^{\|}$thermal fluctuations would destroy the in-plane magnetization, resulting in a phase diagram similar to Fig. 4.

Considering now a thin film with a finite number $n$ of atomic layers, the anisotropies have to be viewed as averages over all layers and can be tuned by varying $n$. A variation of the film thickness thus refers to a certain trajectory ("anisotropy flow" 49 ) in the corresponding phase diagram, e.g., Fig. 5. In this way we can in principle describe reorientation transitions as a function of film thickness.

Finally, we consider the effect of a finite magnetic field. We keep the absolute value of the field constant, $B / J=0.01$, and change only its direction. For simplicity we restrict ourselves to the case $K_{4}^{\|}=0$, and consider the case $S=2$ and $T / J=1$ as an example. The magnetic field is rotated in the $x z$ plane from $\theta=0$ to $\theta=\pi$, where $\theta$ is the angle between the field and the film normal. Figure 6(a) shows the phase diagram for the case $K_{4}^{\perp}=0$. The magnetization tries to be aligned parallel to the magnetic field, but is hindered by the anisotropies. For a sufficiently small $K_{2}$ the magnetization follows the field continuously, whereas for $K_{2}$ larger than a critical value $K_{2 c}$ it exhibits a jump. This behavior is illustrated by the inset in Fig. 6(a), which shows the perpendicular magnetization component as a function of $\theta$ for $K_{2}=0$, $K_{2}=K_{2 c}$, and $K_{2}=2 K_{2 c}$. A negative value of $K_{4}^{\perp}$ does not change this behavior, except that the coexistence regions becomes more narrow. For $K_{4}^{\perp}>0$, however, the first-order line extends to smaller $K_{2}$ and splits into a two-prong fork, as shown in Fig. 6(b). Each prong ends in a critical point. The metastability regions are also indicated in Fig. 6(b). The fork-like feature grows for increasing $K_{4}^{\perp}$ and the critical points even enter the negative $K_{2}$ region for sufficiently large $K_{4}^{\perp}$. As a side remark, in the language of catastrophy theory the phase diagram in the $\left(K_{2}, K_{4}^{\perp}, \theta\right)$ space shown in Fig. 6 can be described by a butterfly catastrophy. The special cut $K_{4}^{\perp}=0$, Fig. 6 (a), shows a cusp.61

\section{B. Effective anisotropies}

The effective magnetic anisotropies are most generally defined through the dependence of the free energy on the magnetization direction. The anisotropic part of the free energy $F(T, \theta, \phi)(\theta$ and $\phi$ being the polar and azimutal angles of the magnetization) depends on the symmetry of the lattice and is uswally written with the help of spherical harmonics $\mathrm{Y}^{m}, 62,40$ or as a series in powers of the direction cosines. 42 For example, we give the expression for tetragonal symmetry, including also the Zeeman term,

$$
\begin{aligned}
F= & -\mathbf{B} \cdot \mathbf{M}-\mathcal{K}_{2} \cos ^{2} \theta-\mathcal{K}_{3} \cos ^{3} \theta-\mathcal{K}_{4}^{\perp} \cos ^{4} \theta \\
& -\mathcal{K}_{4}^{\|} \sin ^{4} \theta(3 / 4+\cos 4 \phi)-\ldots
\end{aligned}
$$

The effective anisotropies $\mathcal{K}_{2}(T)$ etc. depend strongly on temperature. They have to be distinguished from the $m i$ croscopic anisotropies $K_{2}$ etc. appearing in the Hamiltonian (5). Experimentally, the effective anisotropies are determined from, e.g., ferromagnetic resonance (FMR) experiments. To compare the microscopic anisotropies calculated at $T=0$ with measurements of the effective anisotropies performed at finite temperatures, the temperature dependence of the latter has to be known. This task will be pursued in the present section by use of the Schwinger boson technique.

The main source of the temperature dependence of the effective anisotropies is the decreasing magnetization $\mathbf{M}(T) .42$ Other sources, such as the population change of spin-orbit splitted energy levels near the Fermi energy, 63 will not be considered here, since the thermal variation due to these effects occurs mainly at higher temperatures. Note that the effective anisotropies depend on microscopic anisotropy terms of various orders, 43 and can even be present at finite temperatures if corresponding microscopic ones are absent from the Hamiltonian 42

For the determination of the effective anisotropies we proceed similarly as in Ref. 42 . In order to turn the magnetization into a direction $(\theta, \phi)$ different from the equilibrium one, we apply an auxiliary magnetic field B. For the magnetization we use the SBMF result $\mathbf{M}_{0}$, Eq. (14). There are infinitely many choices of the magnetic field $\mathbf{B}$ that result in the same magnetization direction. This freedom of choice introduces some arbitrariness, since the resulting effective anisotropies depend on the choice of $\mathbf{B}$. We here choose the field $\mathbf{B}=(B, 0,0)$ along the $x$ axis and vary only its strength. 42 We insert the SBMF result for the free energy $F_{0}$, Eq. (10), into Eq. (23). To obtain the effective anisotropies we calculate the angles $\theta, \phi$ and the free energy parametrically for a sufficient number of values of the magnetic-field strength $B$ and fit Eq. (23) to the resulting data.

We now apply this method to a system with uniaxial easy-axis anisotropy. In this case the free energy does not depend on the angle $\phi$ in the absence of a magnetic field and hence the effective anisotropies involving $\phi$ vanish. Then the free energy has the form 


$$
F_{0}+\mathbf{B} \cdot \mathbf{M}=-\sum_{n=2}^{\infty} \mathcal{K}_{n} \cos ^{n} \theta
$$

We calculate the free energy $F_{0}$ for equally spaced values of the direction $\operatorname{cosine} \cos \theta$ by varying the field strength $B$. If a first-order reorientation transition takes place at some field value $B_{\text {reo }}$ we use only fields $B \leq B_{\text {reo }}$. We finally obtain the effective anisotropies by a leastsquare fit. Figure 7 shows the effective anisotropies for a thin film with $S=2, J=100$, and uniaxial anisotropies $K_{2} / J=0.02, K_{4}^{\perp} / J=0.01$ (filled symbols) and $K_{2} / J=0.02, K_{4}^{\perp} / J=-0.01$ (open symbols). We here consider relatively large anisotropies for illustrative purposes so that higher-order anisotropies do not vanish in the numerical errors. The odd effective anisotropies $\mathcal{K}_{3}$ etc. vanish because of symmetry. A small sixth-order effective anisotropy $\mathcal{K}_{6}(T)$ appears at finite temperatures, as well as anisotropies of even higher order, which are much smaller, although no corresponding terms are present in the Hamiltonian. The exact values of $\mathcal{K}_{2,4}(0)$ for $T \rightarrow 0$ are $\mathcal{K}_{2}(0)=K_{2} S(S-1 / 2)=$ $\tilde{K}_{2} S^{2}$ and $\mathcal{K}_{4}(0)=K_{4}^{\perp} S(S-1 / 2)(S-1)(S-3 / 2)=$ $\tilde{K}_{4}^{\perp} S^{4}, 42,40$. 2 cf. Eqs. (8) and (9). Our approach reproduces these limiting values. Note that the special generalization of the anisotropy terms from $\mathrm{SU}(2)$ to $\mathrm{SU}(N)$ discussed in App. A is required to obtain these results. For $T \rightarrow T_{c}$ the effective anisotropies vanish as $\mathcal{K}_{n}(T) \propto M^{n}(T) 62.45$ Therefore, $\mathcal{K}_{4}(T)$ decreases faster with increasing temperature than $\mathcal{K}_{2}(T)$ and exhibits a more smooth behavior near $T_{c}$. The effective anisotropies shown in Fig. 月 qualitatively agree with many-body Green's-function results.35

Finally, we emphasize that the calculation of effective anisotropies with this method suffers from ambiguities due to the freedom of choice of the magnetic field and the particular fitting procedure employed. Furthermore, this derivation employs a static calculation of the magnetization and free energy in order to describe a dynamical (FMR) experiment. These problems are common to all calculations of effective anisotropies we are aware of. A full quantum-mechanical, dynamical theory of FMR would be very useful. One possible approach would be to incorporate spin dynamics into the Schwinger boson theory.

\section{CONCLUSIONS AND OUTLOOK}

In summary, we have presented the Schwinger boson mean-field theory for anisotropic 2D ferromagnets, using two different bosonizations. In this study we have focused on the theoretical treatment of single-ion anisotropies of a Heisenberg-type Hamiltonian. The anisotropy energies break the full $\mathrm{SU}(2)$ spin symmetry, leading to a lower residual symmetry, e.g., of Ising or $X Y$ type. We have shown that the results for the magnetization satisfy the Mermin-Wagner theorem 8 also in these cases, in that a finite spontaneous magnetization is only found if the ground state is not continuously degenerate. The theory can describe the various magnetic order-disorder transitions as well as the reorientation transitions found in experiment. The bosonization method can serve as an alternative technique for quantum spin systems, besides the many-body Green'sfunction approach.27 Our results for magnetization and effective anisotropies qualitatively agree with the ones obtained by the latter method. Microscopic calculations of effective anisotropies as functions of temperature are important, since they - and not the microscopic anisotropies appearing in the Hamiltonian - are obtained from experiments. However, the absolute value of the critical temperature at which the spontaneous magnetization becomes finite strongly depends on the details of the theory. Compared to the Green's-function method, Schwinger boson theory has the advantage of treating any spin value $S$ and any orientation of the magnetization relative to the anisotropy axes and the external magnetic field on the same footing. It is also numerically much less demanding. Other types of anisotropies, for example of hexagonal surfaces, can be incorporated in a straightforward manner. Finally, Schwinger boson mean-field theory is a well-controlled approximation in the sense that fluctuations about the equilibrium solution can be treated systematically within a $1 / N$ expansion.

We conclude with enumerating possible generalizations of the theory that are of particular interest for a realistic description of magnetic thin films: The first is the explicit description of thin films of several layers, where the exchange couplings, the magnetic moments, and the anisotropies are microscopic quantities which depend on the layer index. This can be done by a straightforward generalization of the theory, where one only has to keep track of additional layer indices. The second is the inclusion of the long-range magnetic dipole interaction. This interaction is an additional source of anisotropy, which acts quite differently from the singleion anisotropies, resulting for instance in the formation of magnetic domains.64 We reiterate that both the effect of several layers and the dipole interaction can be treated in the present theory as it is, albeit only in a mean-field manner. The third interesting generalization is the inclusion of magnetic dynamics. This would allow to describe the precession of a non-equilibrium magnetization and constitute a microscopic theory of ferromagnetic resonance. We leave these questions as projects for the future.

\section{ACKNOWLEDGMENTS}

We would like to thank K.H. Bennemann for helpful remarks. We gratefully acknowledge financial support by the Deutsche Forschungsgemeinschaft through Sonderforschungsbereich 290, TP A1. 


\section{APPENDIX A:}

In this appendix we present the Schwinger boson theory for anisotropic ferromagnetic films in some more detail. To derive the mean-field equations we start from the Hamiltonian (5). We replace the spin operators at each lattice site by two Bose fields $b_{\uparrow i}$ and $b_{\downarrow i}$ according to Eqs. (1)-(3). The fields $b_{\uparrow i}$ and $b_{\downarrow i}$ have to satisfy the constraint (4) at each lattice site. With the matrix

$$
\mathrm{S}(i) \equiv\left(\begin{array}{cc}
b_{\uparrow i}^{\dagger} b_{\uparrow i} & b_{\uparrow i}^{\dagger} b_{\downarrow i} \\
b_{\downarrow i}^{\dagger} b_{\uparrow i} & b_{\downarrow i}^{\dagger} b_{\downarrow i}
\end{array}\right)
$$

the Hamiltonian reads, up to an additive constant,

$$
\begin{aligned}
H= & -\frac{J}{2} \sum_{\langle i j\rangle} S_{\beta}^{\alpha}(i) S_{\alpha}^{\beta}(j)-\frac{\mathbf{B}}{2} \sum_{i} \boldsymbol{\sigma}_{\beta}^{\alpha} S_{\alpha}^{\beta}(i) \\
& -\frac{K_{2}}{4} \sum_{i}\left[\left(\sigma^{z}\right)_{\beta}^{\alpha} S_{\alpha}^{\beta}(i)\right]^{2}-\frac{K_{4}^{\perp}}{8} \sum_{i}\left[\left(\sigma^{z}\right)_{\beta}^{\alpha} S_{\alpha}^{\beta}(i)\right]^{4} \\
& -\frac{K_{4}^{\|}}{8} \sum_{i}\left(\left[\left(\sigma^{x}\right)_{\beta}^{\alpha} S_{\alpha}^{\beta}(i)\right]^{4}+\left[\left(\sigma^{y}\right)_{\beta}^{\alpha} S_{\alpha}^{\beta}(i)\right]^{4}\right)
\end{aligned}
$$

with the Pauli matrices $\boldsymbol{\sigma}=\left(\sigma^{x}, \sigma^{y}, \sigma^{z}\right)$. Summation over repeated greek indices is implied.

This expression is now generalized to $N$ Bose fields $b_{\alpha}$. Then $\mathrm{S}$ becomes an $N \times N$ matrix of operators $b_{\alpha}^{\dagger} b_{\beta}$ and the constraint is generalized to

$$
b_{\alpha}^{\dagger} b_{\alpha}=N S
$$

The Pauli matrices are generalized to $N \times N$ blockdiagonal matrices with the original Pauli matrices repeated along the diagonal. We keep the notation $\sigma^{z}$ etc.

There is some freedom in the generalization of the Hamiltonian to $N$ boson flavors - we mainly have to make sure that the correct $N=2$ case is recovered. If we naively generalize the second-order anisotropy term as $-\left(K_{2} / 2 N\right)\left[\sum_{\alpha \beta}\left(\sigma^{z}\right)_{\beta}^{\alpha} S_{\alpha}^{\beta}\right]^{2}$, we run into problems, which are most easily illustrated for the case $S=1 / 2$. For $N=2$ the operator $S^{z}$ has the eigenvalues $\pm 1 / 2$ so that $\left(S^{z}\right)^{2}$ only has the eigenvalue $1 / 4$. This is, of course, the reason why the $K_{2}$ term is irrelevant for $S=1 / 2$. However, for $S=1 / 2$ and $N>2(N$ even) we find $N / 2+1$ different eigenvalues of the $z$ spin component $\left(\sigma^{z}\right)_{\beta}^{\alpha} S_{\alpha}^{\beta}$ and $N / 4+1$ (or $N / 4+1 / 2$ ) different eigenvalues of the $K_{2}$ term for $N$ (not) an integer multiple of 4 . Thus in the limit $N \rightarrow \infty$ the second-order anisotropy term has infinitely many different eigenvalues even for $S=1 / 2$, which looks like a semi-classical approximation. Clearly, the mean-field results would depend on $K_{2}$ for $S=1 / 2$, which is incorrect. To remedy this problem we introduce an $N$-dependent anisotropy $K_{2, N}$ such that the energy difference between the two lowest energy states of the second-order anisotropy term,

$$
\Delta e=\frac{N K_{2, N}}{2}\left[S^{2}-(S-2 / N)^{2}\right]=2 K_{2, N} S-\frac{2 K_{2, N}}{N}
$$

is kept constant for all $N$. In particular, $\Delta e=0$ for $S=1 / 2$ and all $N$. This is achieved by setting

$$
K_{2, N}=\frac{S-1 / 2}{S-1 / N} K_{2}
$$

In the mean-field approximation, $N \rightarrow \infty$, we get 55

$$
\tilde{K}_{2} \equiv K_{2, \infty}=K_{2}(S-1 / 2) / S
$$

Analogously, the $N$-dependent fourth-order anisotropies are obtained,

$$
K_{4, N}^{\perp, \|}=\frac{(S-1 / 2)(S-1)(S-3 / 2)}{(S-1 / N)(S-2 / N)(S-3 / N)} K_{4}^{\perp, \|}
$$

and 55

$$
\tilde{K}_{4}^{\perp, \|} \equiv K_{4, \infty}^{\perp, \|}=K_{4}^{\perp, \|}(S-1 / 2)(S-1)(S-3 / 2) / S^{3} .
$$

The fourth-order anisotropy contributions now correctly vanish for $S<2$ even at the mean-field level.

In order to write down a functional integral over the Bose fields, we have to normal order the bosonic operators. The exchange term is trivial, since the operators at different lattices sites commute. By commuting the operators in the anisotropy terms, using the constraint (A3), and dropping a constant we obtain

$$
\begin{aligned}
& H_{\mathrm{SU}(N)}=-\frac{J}{N} \sum_{\langle i j\rangle} S_{\beta}^{\alpha}(i) S_{\alpha}^{\beta}(j)-\frac{\mathbf{B}}{2} \sum_{i} \boldsymbol{\sigma}_{\beta}^{\alpha} S_{\alpha}^{\beta}(i) \\
& -\frac{K_{2, N}^{\prime}}{2 N} \sum_{i}:\left[\left(\sigma^{z}\right)_{\beta}^{\alpha} S_{\alpha}^{\beta}(i)\right]^{2}: \\
& -\frac{K_{4, N}^{\perp}}{2 N^{3}} \sum_{i}:\left[\left(\sigma^{z}\right)_{\beta}^{\alpha} S_{\alpha}^{\beta}(i)\right]^{4}: \\
& -\frac{K_{4, N}^{\|}}{2 N^{3}} \sum_{i}:\left(\left[\left(\sigma^{x}\right)_{\beta}^{\alpha} S_{\alpha}^{\beta}(i)\right]^{4}+\left[\left(\sigma^{y}\right)_{\beta}^{\alpha} S_{\alpha}^{\beta}(i)\right]^{4}\right):
\end{aligned}
$$

where : : denote normal ordering. The second-order anisotropy has obtained a contribution from commuting the bosons in the fourth-order terms, similar to other approaches, 45.42

$$
K_{2, N}^{\prime}=K_{2, N}+\frac{6 N S+4}{N^{2}} K_{4, N}^{\perp}-\frac{6 N S+4}{N^{2}} K_{4, N}^{\|} .
$$

If we want to do a consistent theory to order zero in $1 / N$ we can just drop the corrections. However, since the prefactors $(6 N S+4) / N^{2}=3 S+1$ are not small for $N=2$ it may be better to keep them. Note also that 
they cancel for cubic symmetry. In the following we omit the prime at $K_{2}$ but keep these points in mind.

Next, we go over to the continuum limit7 and write down the functional integral for the partition function. The constraint A3 is implemented using a Lagrange multiplier $\lambda(\mathbf{r}, \tau)$ at each point in space $\mathbf{r}$ and imaginary time $\tau$. $\lambda$ is to be integrated from $-i \infty$ to $+i \infty$ but we can deform the path of integration between these limits. The path of integration has to cross the real axis on the positive side to avoid a branch cut. The partition function reads

$$
Z=\int D^{2} b_{\alpha} D \lambda \exp \left(-\frac{1}{\hbar} \int_{0}^{\hbar \beta} d \tau \int d^{2} r \mathcal{L}[b ; \lambda]\right),
$$

where now $b_{\alpha}(\mathbf{r}, \tau)$ is a complex field. The Lagrangian is

$$
\begin{aligned}
& \mathcal{L}[b ; \lambda]=\frac{\hbar}{a^{2}} b_{\alpha}^{*} \partial_{\tau} b_{\alpha}+J S\left(\partial_{j} b_{\alpha}^{*}\right)\left(\partial_{j} b_{\alpha}\right) \\
& -\frac{J}{N} b_{\alpha}^{*}\left(\partial_{j} b_{\beta}^{*}\right) b_{\beta}\left(\partial_{j} b_{\alpha}\right)-\frac{\mathbf{B}}{2 a^{2}} \boldsymbol{\sigma}_{\beta}^{\alpha} b_{\beta}^{*} b_{\alpha} \\
& \quad-\frac{K_{2, N}}{2 N a^{2}}\left[\left(\sigma^{z}\right)_{\beta}^{\alpha} b_{\beta}^{*} b_{\alpha}\right]^{2}-\frac{K_{4, N}^{\perp}}{2 N^{3} a^{2}}\left[\left(\sigma^{z}\right)_{\beta}^{\alpha} b_{\beta}^{*} b_{\alpha}\right]^{4} \\
& \quad-\frac{K_{4, N}^{\|}}{2 N^{3} a^{2}}\left(\left[\left(\sigma^{x}\right)_{\beta}^{\alpha} b_{\beta}^{*} b_{\alpha}\right]^{4}+\left[\left(\sigma^{y}\right)_{\beta}^{\alpha} b_{\beta}^{*} b_{\alpha}\right]^{4}\right) \\
& +\lambda b_{\alpha}^{*} b_{\alpha}-N S \lambda .
\end{aligned}
$$

Here, $a$ is the lattice spacing, $\partial_{\tau}$ is the time derivative, and $\partial_{j}$ is a spatial derivative along the $j$ direction. Summation over repeated indices is implied.

In the next step, the terms containing more than two $b_{\alpha}$ fields are decoupled using Hubbard-Stratonovich transformations, thereby introducing additional auxiliary fields $\mathbf{Q}, \mathbf{P}$, and $\mathbf{R}$, which have to be integrated over in the functional integral. In a short-hand notation the decoupling of the exchange term may be writtent

$$
\begin{gathered}
-\frac{J}{N} b_{\alpha}^{*}\left(\partial_{j} b_{\beta}^{*}\right) b_{\beta}\left(\partial_{j} b_{\alpha}\right) \rightarrow N J Q_{j} Q_{j}+i J Q_{j} b_{\alpha}^{*}\left(\partial_{j} b_{\alpha}\right) \\
-i J Q_{j}\left(\partial_{j} b_{\alpha}^{*}\right) b_{\alpha} .
\end{gathered}
$$

The $K_{4}^{\perp}$ term contains eight Bose operators, which are decoupled into four according to

$$
\begin{gathered}
-\frac{K_{4, N}^{\perp}}{2 N^{3}}\left[\left(\sigma^{z}\right)_{\beta}^{\alpha} b_{\beta}^{*} b_{\alpha}\right]^{4} \rightarrow \frac{N K_{4, N}^{\perp}}{2} R_{z}^{2} \\
-\frac{K_{4, N}^{\perp}}{N} R_{z}\left[\left(\sigma^{z}\right)_{\beta}^{\alpha} b_{\beta}^{*} b_{\alpha}\right]^{2} .
\end{gathered}
$$

To make the transformation well defined, $R_{z}$ is to be integrated from $-\infty$ to $+\infty$ if $K_{4}^{\perp}>0$ but from $-i \infty$ to $+i \infty$ if $K_{4}^{\perp}<0$. The remaining four-boson term is grouped together with the $K_{2}$ term and decoupled,

$$
\begin{aligned}
-\frac{K_{2, N}+2 K_{4, N}^{\perp} R_{z}}{2 N}\left[\left(\sigma^{z}\right)_{\beta}^{\alpha} b_{\beta}^{*} b_{\alpha}\right]^{2} & \\
\rightarrow & \frac{N\left(K_{2, N}+2 K_{4, N}^{\perp} R_{z}\right)}{2} P_{z}^{2} \\
& -\left(K_{2, N}+2 K_{4, N}^{\perp} R_{z}\right) P_{z}\left(\sigma^{z}\right)_{\beta}^{\alpha} b_{\beta}^{*} b_{\alpha} .
\end{aligned}
$$

Here, the direction of integration of $P_{z}$ must be chosen such that $\left(K_{2, N}+2 K_{4, N}^{\perp} R_{z}\right) P_{z}^{2}$ is real and positive at the end points. The decoupling of the $K_{4}^{\|}$terms is done similarly, introducing additional fields $R_{x, y}$ and $P_{x, y}$.

Up to this point no approximation, apart from the continuum limit, has been made. Now we consider the meanfield approximation for this system. This is exact for $N \rightarrow \infty$ but serves as a lowest-order approximation for $N=2$. The mean-field theory is defined by all auxiliary fields being constant in time. Since the system is ferromagnetic, we assume that they are constant in space as well and write them as $\bar{\lambda}, \overline{\mathbf{Q}}, \overline{\mathbf{P}}$, and $\overline{\mathbf{R}}$. Then, the action is bilinear in the fields $b_{\alpha}$. Written in terms of Fourier transforms the mean-field partition function reads

$$
Z_{0}=Z_{c} \int \prod_{\alpha, \mathbf{k}, i \omega_{n}} d^{2} b_{\alpha}\left(\mathbf{k}, i \omega_{n}\right) \exp \left(-\int d^{2} k \sum_{i \omega_{n}} \mathcal{L}_{0}[b]\right)
$$

with the $b_{\alpha}$-independent part

$$
\begin{aligned}
Z_{c}= & \exp \left(-\mathcal{N} N \beta J \overline{\mathbf{Q}} \cdot \overline{\mathbf{Q}} a^{2}+\mathcal{N} a^{2} N S \beta \bar{\lambda}\right. \\
& -\mathcal{N} N \frac{\beta \tilde{K}_{2}}{2} \bar{P}_{z}^{2}-\mathcal{N} N \frac{\beta \tilde{K}_{4}^{\perp}}{2} \bar{R}_{z}^{2} \\
& -\mathcal{N} N \beta \tilde{K}_{4}^{\perp} \bar{R}_{z} \bar{P}_{z}^{2}-\mathcal{N} N \frac{\beta \tilde{K}_{4}^{\|}}{2}\left[\bar{R}_{x}^{2}+\bar{R}_{y}^{2}\right] \\
& \left.-\mathcal{N} N \beta \tilde{K}_{4}^{\|} \times\left[\bar{R}_{x} \bar{P}_{x}^{2}+\bar{R}_{y} \bar{P}_{y}^{2}\right]\right),
\end{aligned}
$$

where $\mathcal{N}$ is the number of sites. The Lagrangian is

$$
\begin{aligned}
& \mathcal{L}_{0}[b]=\beta a^{2} \sum_{\alpha}\left(-i \hbar \omega_{n}+J S k^{2} a^{2}-\frac{\beta J}{S} \overline{\mathbf{Q}} \cdot \overline{\mathbf{Q}} a^{2}\right. \\
& \left.+a^{2} \bar{\lambda}\right) b_{\alpha}^{*}\left(\mathbf{k}, i \omega_{n}\right) b_{\alpha}\left(\mathbf{k}, i \omega_{n}\right) \\
& \quad-\beta a^{2} \sum_{\alpha \beta}\left(\frac{\mathbf{B}}{2} \cdot \sigma_{\beta}^{\alpha}+\tilde{K}_{2} \bar{P}_{z}\left(\sigma^{z}\right)_{\beta}^{\alpha}\right. \\
& \quad+2 \tilde{K}_{4}^{\perp} \bar{R}_{z} \bar{P}_{z}\left(\sigma^{z}\right)_{\beta}^{\alpha} \\
& \left.\quad+2 \tilde{K}_{4}^{\|}\left[\bar{R}_{x} \bar{P}_{x}\left(\sigma^{x}\right)_{\beta}^{\alpha}+\bar{R}_{y} \bar{P}_{y}\left(\sigma^{y}\right)_{\beta}^{\alpha}\right]\right) b_{\beta}^{*} b_{\alpha} .
\end{aligned}
$$

After integrating out the $b_{\alpha}$ fields and performing the sum over field indices $\alpha$, we obtain, up to an irrelevant constant factor,

$$
Z_{0}=Z_{c} \exp \left(-\frac{N}{2} \frac{\mathcal{N} a^{2}}{4 \pi^{2}} \int d^{2} k \sum_{i \omega_{n}} \ln \operatorname{det} \mathrm{M}\left(\mathbf{k}, i \omega_{n}\right)\right)
$$

with the $2 \times 2$ matrix

$$
\begin{aligned}
\mathrm{M}= & \left(-i \beta \hbar \omega_{n}+\beta J S k^{2} a^{2}+\bar{\Lambda}\right) \mathbf{1}-\frac{\beta \mathbf{B}}{2} \cdot \boldsymbol{\sigma} \\
& -\beta \tilde{K}_{2} \bar{P}_{z} \sigma^{z}-2 \beta \tilde{K}_{4}^{\perp} \bar{R}_{z} \bar{P}_{z} \sigma^{z} \\
& -2 \beta \tilde{K}_{4}^{\|}\left(\bar{R}_{x} \bar{P}_{x} \sigma^{x}+\bar{R}_{y} \bar{P}_{y} \sigma^{y}\right) .
\end{aligned}
$$


Note that $Z_{0}$ only depends on $\bar{\lambda}$ and $\overline{\mathbf{Q}}$ through

$$
\bar{\Lambda} \equiv a^{2} \beta \bar{\lambda}-\frac{\beta J}{S} \overline{\mathbf{Q}} \cdot \overline{\mathbf{Q}} a^{2},
$$

just as in the isotropic case. 7 Evaluation of the frequency sum and momentum integral in Eq. (A19) yields the free energy per boson

$$
\begin{aligned}
\beta F_{0} \equiv- & \frac{1}{\mathcal{N} N} \ln Z_{0} \\
=- & S \bar{\Lambda}+\frac{\beta \tilde{K}_{2}}{2} \bar{P}_{z}^{2}+\frac{\beta \tilde{K}_{4}^{\perp}}{2} \bar{R}_{z}^{2} \\
& +\beta \tilde{K}_{4}^{\perp} \bar{R}_{z} \bar{P}_{z}^{2}+\frac{\beta \tilde{K}_{4}^{\|}}{2}\left(\bar{R}_{x}^{2}+\bar{R}_{y}^{2}\right) \\
& +\beta \tilde{K}_{4}^{\|}\left(\bar{R}_{x} \bar{P}_{x}^{2}+\bar{R}_{y} \bar{P}_{y}^{2}\right) \\
& +\frac{1}{8 \pi \beta J S}\left[\operatorname{diln}\left(1-e^{-\bar{\Lambda}+\beta B^{\prime} / 2}\right)\right. \\
& \left.+\operatorname{diln}\left(1-e^{-\bar{\Lambda}-\beta B^{\prime} / 2}\right)\right]
\end{aligned}
$$

with $B^{\prime}=\left|\mathbf{B}^{\prime}\right|$ and

$$
\begin{aligned}
B_{x}^{\prime} & =B_{x}+4 \tilde{K}_{4}^{\|} \bar{R}_{x} \bar{P}_{x}, \\
B_{y}^{\prime} & =B_{y}+4 \tilde{K}_{4}^{\|} \bar{R}_{y} \bar{P}_{y}, \\
B_{z}^{\prime} & =B_{z}+2 \tilde{K}_{2} \bar{P}_{z}+4 \tilde{K}_{4}^{\perp} \bar{R}_{z} \bar{P}_{z} .
\end{aligned}
$$

The dilogarithm function is defined by the series representation

$$
\operatorname{diln} x \equiv-\sum_{n=1}^{\infty} \frac{(1-x)^{n}}{n^{2}} .
$$

We are mainly interested in the magnetization

$$
\begin{aligned}
\mathbf{M}_{0} & \equiv-2 \frac{d F_{0}}{d \mathbf{B}}=-2 \frac{\partial F_{0}}{\partial \mathbf{B}}=-\frac{1}{8 \pi \beta J S} \frac{\mathbf{B}^{\prime}}{B^{\prime}} \\
\times & {\left[\ln \left(1-e^{-\bar{\Lambda}+\beta B^{\prime} / 2}\right)-\ln \left(1-e^{-\bar{\Lambda}-\beta B^{\prime} / 2}\right)\right] . }
\end{aligned}
$$

We have used that the derivatives of $F_{0}$ with respect to $\bar{\Lambda}$ and $\overline{\mathbf{P}}$ vanish at the saddle point. A factor of 2 appears, since in the physical system two bosons make up one spin. For the isotropic case $\mathbf{B}^{\prime}$ equals $\mathbf{B}$ and we recover the results of Refs. 6 and 7 .

Finally, we have to determine the auxiliary fields by finding the lowest stable free-energy saddle point. We set the derivatives of $\ln Z_{0}$ with respect to $\bar{\Lambda}, \overline{\mathbf{P}}$, and $\overline{\mathbf{R}}$ to zero and solve the resulting equations simultaneously. From $\partial \ln Z_{0} / \partial \bar{\Lambda}=0$ we obtain the saddle-point equation

$$
\begin{aligned}
S= & -\frac{1}{8 \pi \beta J S}\left[\ln \left(1-e^{-\bar{\Lambda}+\beta B^{\prime} / 2}\right)\right. \\
& \left.+\ln \left(1-e^{-\bar{\Lambda}-\beta B^{\prime} / 2}\right)\right],
\end{aligned}
$$

which can be analytically solved for $\Lambda$. By taking the derivatives with respect to the other auxiliary fields one finds that for all saddle-point solutions

$$
\bar{R}_{i}=\bar{P}_{i}^{2}, \quad i=x, y, z .
$$

This equation either follows directly from the saddlepoint equations or, for special values of $\bar{R}_{i}$, the free energy is found to be independent of the corresponding $\bar{P}_{i}$ so that we can choose $\bar{P}_{i}$ to satisfy Eq. (A29). We end up with a free energy which depends on the four auxiliary parameters $\bar{\Lambda}$ and $\overline{\mathbf{P}}$,

$$
\begin{aligned}
\beta F_{0}= & -S \bar{\Lambda}+\frac{\beta \tilde{K}_{2}}{2} \bar{P}_{z}^{2} \\
& +\frac{3 \beta \tilde{K}_{4}^{\perp}}{2} \bar{P}_{z}^{4}+\frac{3 \beta \tilde{K}_{4}^{\|}}{2}\left(\bar{P}_{x}^{4}+\bar{P}_{y}^{4}\right) \\
& +\frac{1}{8 \pi \beta J S}\left[\operatorname{diln}\left(1-e^{-\bar{\Lambda}+\beta B^{\prime} / 2}\right)\right. \\
& \left.+\operatorname{diln}\left(1-e^{-\bar{\Lambda}-\beta B^{\prime} / 2}\right)\right]
\end{aligned}
$$

with

$$
\begin{aligned}
& B_{x}^{\prime}=B_{x}+4 \tilde{K}_{4}^{\|} \bar{P}_{x}^{3}, \\
& B_{y}^{\prime}=B_{y}+4 \tilde{K}_{4}^{\|} \bar{P}_{y}^{3}, \\
& B_{z}^{\prime}=B_{z}+2 \tilde{K}_{2} \bar{P}_{z}+4 \tilde{K}_{4}^{\perp} \bar{P}_{z}^{3} .
\end{aligned}
$$

In the uniaxial case $K_{4}^{\|}=0$ there are only two relevant parameters, $\bar{\Lambda}$ and $\bar{P}_{z}$.

By solving the saddle-point equations $\partial \ln Z_{0} / \partial \bar{P}_{i}=0$ we find that there are typically two solutions for each component $\bar{P}_{i}$. One is $\bar{P}_{i}=M_{0, i}$, i.e., $\bar{P}_{i}$ is the corresponding magnetization component, and the other is given by $B_{i}^{\prime}=B_{i}$. For example, in the uniaxial case, $K_{4}^{\|}=0\left(\tilde{K}_{4}^{\|}=0\right)$ the free energy does not depend on $\bar{P}_{x}$ and $\bar{P}_{y}$ whereas $M_{0, x}$ and $M_{0, y}$ have well-defined values, which need not be zero in the presence of an external field. Thus $\bar{P}_{x, y}$ cannot equal $M_{0, x, y}$. The saddlepoint equations are solved by the second type of solution, $B_{x, y}^{\prime}=B_{x, y}$, since $K_{4}^{\perp}=0$. On the other hand, the $z$ component is $\bar{P}_{z}=M_{0, z}$.

It turns out that the relevant saddle point of the free energy $F_{0}$ is not a minimum with respect to all four parameters. For example, it is always a maximum with respect to $\bar{\Lambda}$ (or $\bar{\lambda}$ ). Intuitively, one would expect $\bar{\Lambda}$ to run off to infinity in this case. However, since $\lambda$ in Eq. (A11) has to be integrated from $-i \infty$ to $+i \infty$, fluctuations in $\lambda$ are along the imaginary direction, $\lambda(\mathbf{r}, \tau)=\bar{\lambda}+i \Delta \lambda(\mathbf{r}, \tau)$, where $\Delta \lambda$ is real. In particular, this holds for global changes of $\bar{\lambda}$. With respect to $\Delta \lambda$, the free energy indeed has a minimum at $\Delta \lambda=0$. For the HubbardStratonovich fields $\overline{\mathbf{P}}$ and $\overline{\mathbf{R}}$ the direction of fluctuations depends on the values of the anisotropy constants. The stability of each saddle point against global changes of the auxiliary parameters has to be checked in order to find the lowest stable one. Note that for positive anisotropy constants the fluctuations of $\overline{\mathbf{P}}$ and $\overline{\mathbf{Q}}$ are purely real and the stability analysis becomes straightforward. 
* Electronic address: timm@physik.fu-berlin.de.

${ }^{1}$ J. Schwinger, Quantum Theory of Angular Momentum, edited by L. Biedenharn and H. Van Dam (Academic, New York, 1965).

${ }^{2}$ N. Read and D.M. Newns, J. Phys. C 16, 3273 (1983).

${ }^{3}$ D.P. Arovas and A. Auerbach, Phys. Rev. B 38, 316 (1988); A. Auerbach and D.P. Arovas, Phys. Rev. Lett. 61, 617 (1988).

${ }^{4}$ A. Auerbach, Interacting Electrons and Quantum Magnetism (Springer, New York, 1994).

${ }^{5}$ O.A. Starykh, Phys. Rev. B 50, 16428 (1994); A.V. Chubukov and O.A. Starykh, ibid. 52, 440 (1995).

${ }^{6}$ N. Read and S. Sachdev, Phys. Rev. Lett. 75, 3509 (1995).

${ }^{7}$ C. Timm, S.M. Girvin, P. Henelius, and A.W. Sandvik, Phys. Rev. B 58, 1464 (1998).

${ }^{8}$ N.D. Mermin and H. Wagner, Phys. Rev. Lett. 17, 1133 (1966).

9 T. Holstein and H. Primakoff, Phys. Rev. 58, 1908 (1940).

${ }^{10}$ D.P. Pappas, K.P. Kämper, and H. Hopster, Phys. Rev. Lett. 64, 3179 (1990).

11 J. Thomassen, F. May, B. Feldmann, M. Wuttig, and H. Ibach, Phys. Rev. Lett. 69, 3831 (1992).

${ }^{12}$ U. Gradmann, Handbook of Magnetic Materials (Elsevier, Amsterdam, 1993), Vol. 7; J.A.C. Bland and B. Heinrich, Ultrathin Magnetic Structures (Springer Verlag, Berlin, 1994).

${ }^{13}$ Z.Q. Qiu, J. Pearson, and S.D. Bader, Phys. Rev. Lett. 70, 1006 (1993); D. Li, M. Freitag, J. Pearson, Z.Q. Qiu, and S.D. Bader, ibid. 72, 3112 (1994).

${ }^{14}$ G. Lugert, W. Robl, L. Pfau, M. Brockmann, and G. Bayreuther, J. Magn. Magn. Mater. 121, 498 (1993).

${ }^{15}$ B. Schulz and K. Baberschke, Phys. Rev. B 50, 13467 (1994); M. Farle, W. Platow, A.N. Anisimov, B. Schulz, and K. Baberschke, J. Magn. Magn. Mater. 165, 74 (1997).

${ }^{16}$ K. Baberschke, Appl. Phys. A 62, 417 (1996); M. Farle, B. Mirwald-Schulz, A.N. Anisimov, W. Platow, and K. Baberschke, Phys. Rev. B 55, 3708 (1997).

${ }^{17}$ R. Allenspach, J. Magn. Magn. Mater. 129, 160 (1994).

18 O. Schulte, F. Klose, and W. Felsch, Phys. Rev. B 52, 6480 (1995).

19 A. Berger and H. Hopster, Phys. Rev. Lett. 76, 519 (1996).

${ }^{20}$ G. Garreau, E. Beaurepaire, K. Ounadjela, and M. Farle, Phys. Rev. B 53, 1083 (1996).

${ }^{21}$ C. Herring and C. Kittel, Phys. Rev. 81, 869 (1951); S.V. Maleev, Sov. Phys. JETP 43, 1240 (1976); V.L. Pokrovsky and M.V. Feigel'man, Sov. Phys. JETP 45, 291 (1977).

22 R.P.Erickson and D.L. Mills, Phys. Rev. B 43, 10715 (1991); 44, 11825 (1991).

23 S.V. Tyablikov, Ukr. Mat. Zh. 11287 (1959).

${ }^{24}$ R. Tahir-Kheli and D. ter Haar, Phys. Rev. 127, 88 (1962).

${ }^{25}$ H.B. Callen, Phys. Rev. 130, 890 (1963).

${ }^{26}$ S.V.Tyablikov, Methods in the quantum theory of magnetism (Plenum Press, New York, 1967).

${ }^{27}$ P. Fröbrich, P.J. Jensen, and P.J. Kuntz, Europ. Phys. J. B 13, 477 (1999).

${ }^{28}$ W. Döring, Z. Naturforsch. A16, 1008 (1961).

${ }^{29}$ W. Haubenreisser, W. Brodkorb, A. Corciovei, and G. Costache, Phys. Stat. Solidi B 53, 9 (1972).

${ }^{30}$ Diep-the-Hung, J.C.S. Levy, and O. Nagai, Phys. Stat. Solidi B 93, 351 (1979).
${ }^{31}$ L.P. Shi and W.G. Yang, J. Phys. Condens. Mater. 4, 7997 (1992).

32 P. Bruno, Phys. Rev. B 43, 6015 (1991).

33 D.K. Morr, P.J. Jensen, and K.H. Bennemann, Surf. Sci. 307-309, 1109 (1994).

${ }^{34}$ P. Henelius, A.W. Sandvik, C. Timm, and S.M. Girvin, Phys. Rev. B 61, 364 (2000).

${ }^{35}$ A. Ecker, P. Fröbrich, P.J. Jensen, and P.J. Kuntz, J. Phys. Cond. Mat. 11, 1557 (1999).

${ }^{36}$ S.B. Haley, Phys. Rev. B 17, 337 (1978).

${ }^{37}$ F.B. Anderson and H.B. Callen, Phys. Rev. 136, A1068 (1964).

38 M.E. Lines, Phys. Rev. 156, 534 (1967).

${ }^{39}$ P.J. Jensen and K.H. Bennemann, Solid State Commun. 100, 585 (1996).

${ }^{40}$ Y. Millev and M. Fähnle, Phys. Rev. B 51, 2937 (1995).

${ }^{41}$ P.J. Jensen and K.H. Bennemann, Solid State Commun. 105, 577 (1998), and references therein.

${ }^{42}$ P.J. Jensen and K.H. Bennemann, Magnetism and Electronic Correlations in Local-Moment Systems: Rare-Earth Elements and Compounds, edited by M. Donath, P.A. Dowben, and W. Nolting (World Scientific, Singapore, 1998), p. 113.

43 A. Moschel and K.D. Usadel, Phys. Rev. B 49, 12868 (1994).

44 A. Hucht and K.D. Usadel, Phys. Rev. B 55, 12309 (1997); Phil. Mag. B 80, 275 (2000).

${ }^{45}$ P.J. Jensen and K.H. Bennemann, Ann. Physik (Leipzig) 2, 475 (1993).

${ }^{46}$ X. Hu and Y. Kawazoe, Phys. Rev. B 54, 65 (1996), and references therein.

${ }^{47}$ P. Politi, A. Rettori, M.G. Pini, and D. Pescia, J. Magn. Magn. Mater. 140-144, 647 (1995); A. Abanov, V. Kalatsky, V.L. Pokrovsky, and W.M. Saslow, Phys. Rev. B 51, 1023 (1995), and references therein.

${ }^{48}$ H.B.G. Casimir, J. Smit, U. Enz, J.F. Fast, H.P.J. Wijn, E.W. Gorter, A.J.W. Duyvesteyn, J.D. Fast, and J.J. de Jong, J. Phys. Radium 20, 360 (1959).

49 Y.T. Millev, H.P. Oepen, and J. Kirschner, Phys. Rev. B 57, 5837 (1998); 57, 5848 (1998).

50 S.T. Chui, Phys. Rev. B 50, 12559 (1994); A. Hucht, A. Moschel, and K.D. Usadel, J. Magn. Magn. Mater. 148, 32 (1995); O. Iglesias, A. Valencia, and A. Labarta, ibid. 196-197, 819 (1999).

51 S.L. Sondhi, A. Karlhede, S.A. Kivelson, and E.H. Rezayi, Phys. Rev. B 47, 16419 (1993).

${ }^{52}$ R. Côté, A.H. MacDonald, L. Brey, H.A. Fertig, S.M. Girvin, and H.T.C. Stoof, Phys. Rev. Lett. 78, 4825 (1997); S.M. Girvin and A.H. MacDonald, in Perspectives in Quantum Hall Effects, edited by S. Das Sarma and A. Pinczuk (Wiley, New York, 1997).

53 M.J. Manfra, E.H. Aifer, B.B. Goldberg, D.A. Broido, L. Pfeiffer, and K. West, Phys. Rev. B 54, R17327 (1996).

54 The dipolar interaction may be important, though. Because of the large external magnetic fields present in quantum Hall experiments, we do not expect the dipolar interaction to lead to the formation of domains. However, it does favour a spin orientation parallel to the $2 \mathrm{D}$ electron gas. This effect may be observable in experiments with magnetic fields tilted away from the normal axis. 
${ }^{55}$ Y. Millev and M. Fähnle, Phys. Rev. B 52, 4336 (1995).

${ }^{56}$ P.J. Jensen (unpublished).

${ }^{57}$ At first glance it may seem surprising that a factor of $S^{2}$ instead of the quantum-mechanical $S(S+1)$ appears in the expressions. However, the factor comes from the exchange interaction, as indicated by the factor $J$. The exchange term contains two spin operators at different sites, which commute. Thus $\mathbf{S}_{i} \cdot \mathbf{S}_{j}=S^{2}$ if $\mathbf{S}_{i} \| \mathbf{S}_{j}$.

${ }^{58}$ P.J. Jensen and K.H. Bennemann, Phys. Rev. B 42, 849 (1990).

59 The true ground state of a perpendicularly magnetized thin film is not a homogeneous one but, due to the long-range dipole coupling, a stripe domain phase with a vanishing net magnetization. By considering this multidomain state the phase diagram of Fig. 3 is altered somewhat. For a thorough discussion of these phases see Ref. 64. In the present work we assume homogeneous phases.

60 The spin quantum number does not affect the qualitative form of the phase diagram, as long as $S \geq 2$ so that fourthorder anisotropies are relevant. We here study $S=2$.

${ }^{61}$ P.T. Saunders, An introduction to catastrophy theory (Cambridge University Press, Cambridge, 1980).

${ }^{62}$ H.B. Callen and E.R. Callen, J. Phys. Chem. Solids 27, 1271 (1966).

63 T.H. Moos, W. Hübner, and K.H. Bennemann, Solid State Commun. 98, 639 (1996).

${ }^{64}$ P.J. Jensen and K.H. Bennemann, Phys. Rev. B 52, 16012 (1995), and references therein.

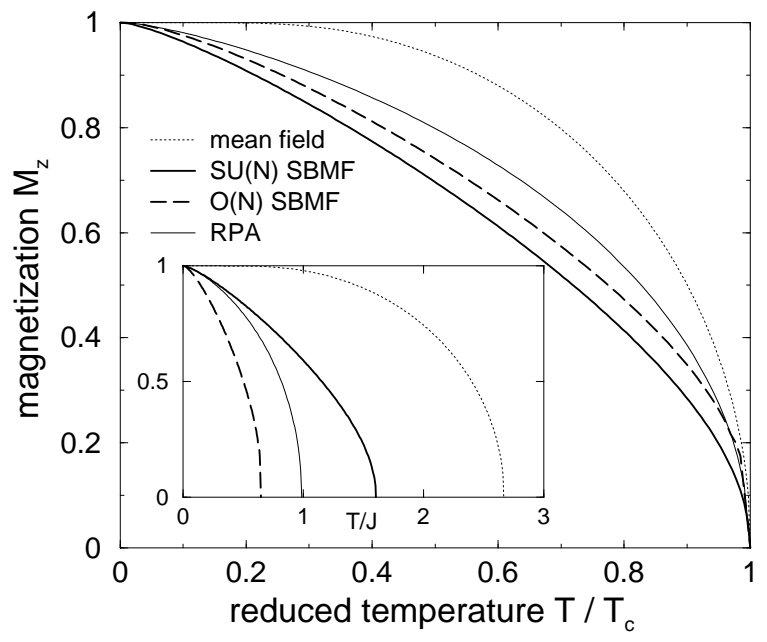

FIG. 1. Magnetization as predicted by various theories as a function of reduced temperature $T / T_{c}$ for a film in a vanishing external magnetic field, $\mathbf{B}=0$, with an easy axis defined by $K_{2} / J=0.01$, spin $S=1$, and no higher-order anisotropies. The magnetic dipole coupling is neglected. The inset shows the magnetization as a function of the absolute temperature $T / J$ in units of exchange $J$. The line denoted by 'RPA' refers to a calculation within a many-body Green's-function approach with Anderson-Callen decoupling of the single-ion anisotropy.27

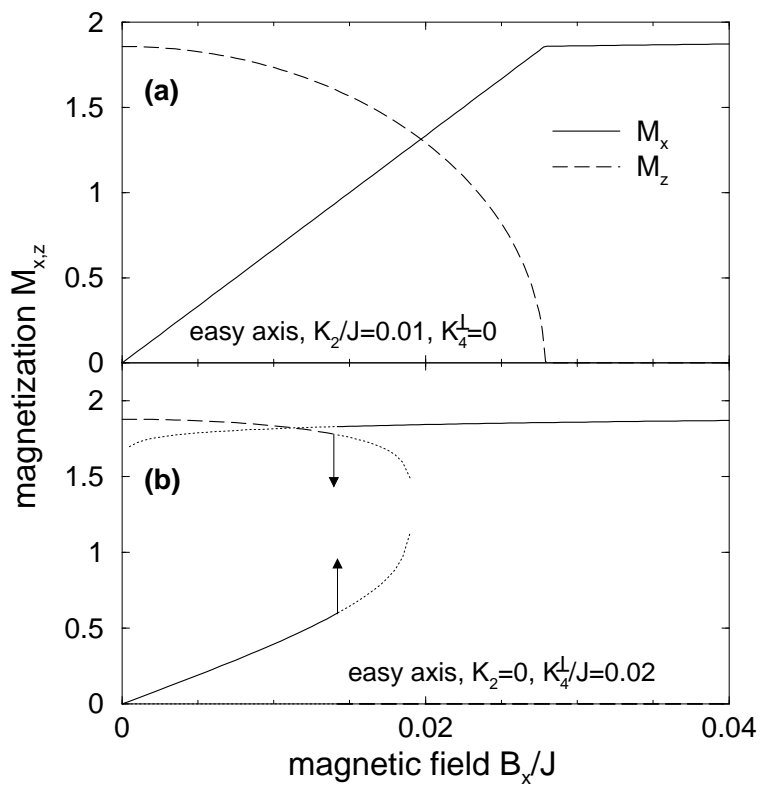

FIG. 2. Reorientation transitions as a function of external magnetic field for a film with exchange $J=100$ and spin $S=2$ at temperature $T / J=1$. (a) Film with second-order easy-axis anisotropy $K_{2} / J=0.01$ and $K_{4}^{\perp}=K_{4}^{\|}=0$. The field is applied in the $x$ direction, i.e., perpendicularly to the easy axis. The solid and dashed lines represent the $x$ and $z$ components of the magnetization. In this case a second-order reorientation transition takes place. (b) The same quantities for a film with forth-order easy-axis anisotropy $K_{4}^{\perp} / J=0.02$ and $K_{2}=K_{4}^{\|}=0$. Here, a first-order reorientation transition takes place, denoted by the arrows. The dotted lines refer to meta-stable states of higher energy. 


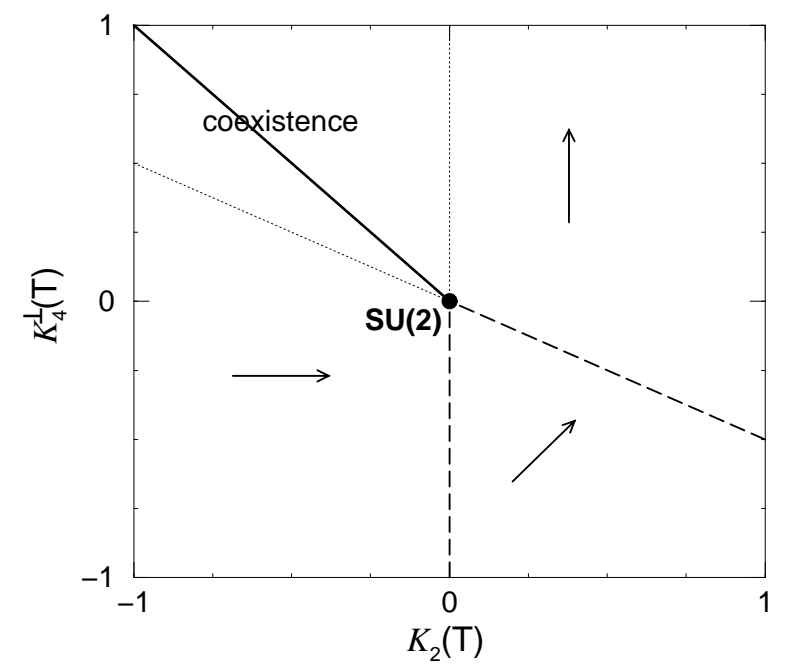

FIG. 3. Phase diagram for the effective anisotropies $\mathcal{K}_{2}(T)$ and $\mathcal{K}_{4}^{\perp}(T)$ for a vanishing external magnetic field und $\mathcal{K}_{4}^{\|}=0.46,16,49$ Three different phases are found, defined by different directions of the magnetization with respect to the film plane (perpendicular, in-plane, canted), indicated by arrows. These phases are separated either by a first order (solid line) or by a second order phase transition (dashed lines), which merge at the tricritical point $\mathcal{K}_{2 c}=\mathcal{K}_{4 c}^{\perp}=0$, corresponding to the isotropic, $\mathrm{SU}(2)$-symmetric case. A coexistence region between the perpendicular and in-plane phases exists near the first order phase transition boundary, indicated by the dotted lines.

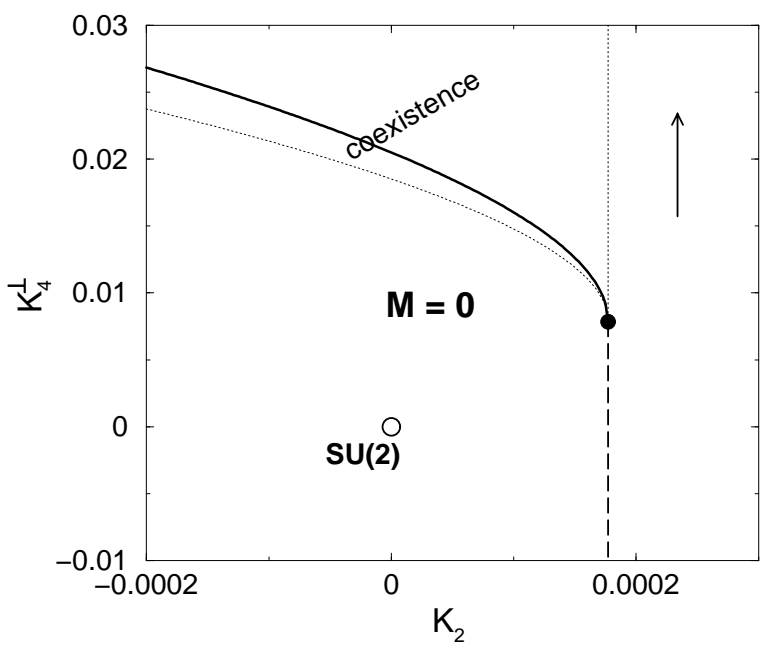

FIG. 4. Phase diagram of a thin film in the $K_{2}-K_{4}^{\perp}$ plane in a vanishing external magnetic field as calculated from SBMF theory. The parameters are spin $S=2$, exchange $J=100$, temperature $T / J=3$, and in-plane anisotropy $K_{4}^{\|}=0$. The phase to the lower left of the phase diagram now exhibits no net magnetization, $\mathbf{M}=0$. The open circle denotes the isotropic, $\mathrm{SU}(2)$-symmetric case $K_{2}=K_{4}^{\perp}=0$. The other symbols are the same as in Fig. 3.



FIG. 5. Phase diagram for a thin film as in Fig. A but with a finite in-plane magnetic anisotropy $K_{4}^{\|} / J \approx 4.3 \times 10^{-4}$, big enough to allow a finite in-plane magnetization at this temperature. The square denotes the point of full cubic symmetry $\left(K_{2}=0, K_{4}^{\perp}=K_{4}^{\|}\right)$. The dotted lines are borders of two coexistence regions. In the left one both perpendicular and in-plane magnetization are metastable, while in the right one perpendicular and canted states are metastable.

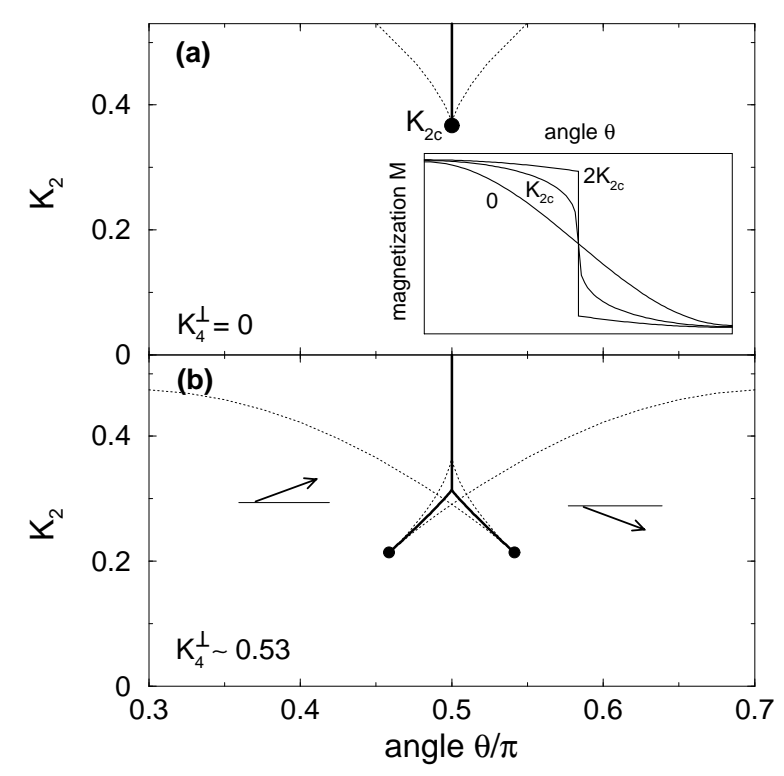

FIG. 6. Phase diagram for a thin film in the $K_{2}-\theta$ plane as calculated from SBMF theory. An easy-axis magnet is assumed, with $K_{2}>0$. A magnetic field of strength $B / J=0.01$ is applied in the $x z$ plane, forming an angle $\theta$ with the film normal. We assume $J=100, S=2, T=J$, and $K_{4}^{\|}=0$. Furthermore, in (a) $K_{4}^{\perp}=0$, whereas in (b) $K_{4}^{\perp} / J \approx 5.3 \times 10^{-3}$. The thick solid lines denote first-order transitions ending in critical points. The dotted lines are the boundaries of coexistence regions. The inset shows the magnetization as a function of $\theta$ for the case (a) with $K_{2}=0, K_{2}=K_{2 c}$, and $K_{2}=2 K_{2 c}, K_{2 c}$. 


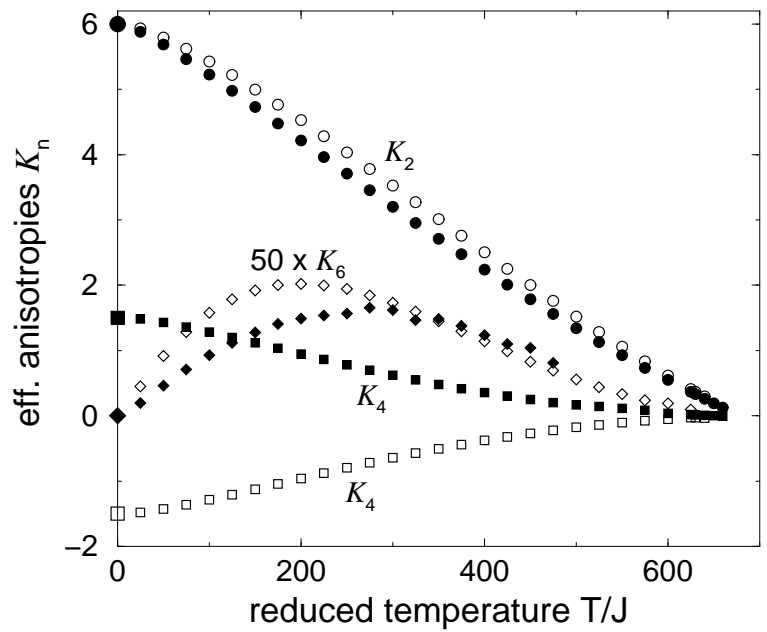

FIG. 7. Effective anisotropies $\mathcal{K}_{2}, \mathcal{K}_{4}$, and $\mathcal{K}_{6}$ as functions of temperature calculated from SBMF theory. A thin film is assumed with $S=2, J=100, K_{2} / J=0.01, K_{4}^{\|}=0$, and $K_{4}^{\perp} / J=10^{-3}$ (filled symbols) and $K_{4}^{\perp} / J=-10^{-3}$ (open symbols). We use rather large microscopic anisotropies to demonstrate more clearly the appearance of a small sixth-order effective anisotropy $\mathcal{K}_{6}$, which is shown scaled by a factor of 50 . The big symbols denote the exact values at $T=0$. 